\title{
P. Lazarević Di Giacomo, M.R. Leto (a cura di), L’Adriatico tra sogno e realtà, Edi- zioni dell'Orso, Alessandria 2019 (= Studi interadriatici, I), pp. 470.
}

Il volume collettaneo L'Adriatico tra sogno e realtà, a cura di Persida Lazarević Di Giacomo e Maria Rita Leto, raccoglie i contributi dell'omonimo convegno internazionale che si è tenuto a ottobre del 2017 all’ Università degli studi “G. d'Annunzio” di Chieti-Pescara. Si tratta di uno sguardo composito e variegato sui nuovi studi, temi e indirizzi d'indagine dedicati a un argomento di cui la slavistica italiana si occupa dai suoi albori - quello del mare Adriatico, del "Mediterraneo minimo" (Ivetic), inesauribile fonte di storia, linguaggi, testi e significati simbolici. Il volume è dedicato alla memoria della collega Dunja Kalođera, prematuramente scomparsa prima dell'uscita di questi atti a cui ha contribuito, testimoniando ancora una volta il suo profondo legame biografico e professionale con il mare delle sue origini.

I testi raccolti sono in lingua italiana, tranne due in inglese; ogni lavoro è corredato da due riassunti in lingue diverse, e il volume contiene i profili degli autori e l'indice dei nomi. I 26 contributi sono suddivisi in 3 sezioni che molto genericamente si potrebbero definire come storica, linguistica e letteraria, anche se la maggior parte dei lavori spazia in ambiti interdisciplinari, toccando problemi e servendosi di strumenti anche di storia culturale, antropologia letteraria, etnologia o studi culturali, motivo per cui nella presente recensione non seguiremo strettamente l'ordine dei contributi proposto dal volume, ma un'altra - una delle possibili - logiche della loro associazione.

Apre il volume il saggio di Egidio Ivetic, dedicato ai modi in cui è possibile intendere la storia e il presente dell'Adriatico come "perfetto caso di mare regione". L'autore indaga sulla possibilità di scrittura di una storia nuova e transnazionale dell'Adriatico, e auspica la creazione di un'identità regionale come alternativa allo status di periferia "che i contesti rivieraschi vivono in riferimento alle proprie culture nazionali”. L'articolo ha un respiro ampio (storico, geografico, culturale, politico, antropologico) e offre una rassegna della vasta letteratura finora scritta sull'argomento, con termini chiave impostati da Braudel e Graciotti. Si tratta di riflessioni che l'autore ha rielaborato più nel dettaglio nel suo libro Storia dell'Adriatico. Un mare e la sua civiltà (Il Mulino, Bologna 2019).

Al campo più strettamente storico appartengono i saggi di Ivan Pederin e Pëllumb Xhufi, che, grazie a indagini archivistiche capillari, mettono in luce nuovi dati su contatti culturali, costumi quotidiani ed episodi militari dell'Adriatico sotto il dominio veneto. Riccamente corredato di immagini tratte dalla Gloria del Ducato di Carniola di Johann Weichard (Janez Vajkard) Valvasor, il contributo di Maria Bidovec si inserisce in queste riflessioni sulla guerra e la pace nell'Adriatico, osservandolo però dalla prospettiva continentale, austriaca del suo autore. Valvasor al mare dedica un ruolo se- 
condario, ma comunque vitale: in senso commerciale e, con ancor maggiore cognizione di causa, in senso militare. Accurate ricerche archivistiche contraddistinguono anche il contributo di Alessandro Cifariello, che espone dettagli di storia ecclesiastica, diplomatica, politica e accademica tra l'Italia postunitaria e il movimento nazionale croato e jugoslavo, in particolare attraverso l'azione del vescovo Strossmayer, che scopriamo nella veste di influente ed abile promotore degli studi slavi in Italia.

Testi agiografici slavo-ecclesiastici sono presi in esame da Maria Chiara Ferro che si occupa del culto di San Nicola di Bari, e in particolare delle narrazioni sulle sue tre apparizioni miracolose al sovrano serbo Stefan Uroš III Nemanjić, detto Dečanski, che collegano eventi accaduti nell'Adriatico con la tradizione scrittoria della Rus'. Di storia della grammatica tratta il contributo di Vesna Deželjin, che analizza il manoscritto della morfologia italiana per apprendenti croatofoni di Dragutin Antun Parčić, ritrovato nel 2004 nell'Archivio vescovile della città di Krk (Veglia), sull'omonima isola. I lavori di Natalia Guseva, Carmela Perta e Loreta Šimunić analizzano fenomeni contemporanei di contatti interlinguistici. Natalia Guseva propone un'analisi contrastiva dell'adattamento morfologico dello sloveno (sull'esempio del resiano) e del croato (sull'esempio del molisano) parlati in Italia, paragonandoli all'erosione linguistica del russo presente tra l'immigrazione russofona, e rilevando importanti analogie di comportamento delle lingue slave nel contatto interlinguistico. Carmela Perta prende in esame varie comunità albanofone nel territorio italiano (tenendo conto di intersezioni di minoranze storiche e quelle di recente immigrazione), utilizzando strumenti sociolinguistici per osservare gli shift motivati da scelte identitarie. Loreta Šimunić infine si muove nel campo intermedio tra il bilinguismo e la linguistica di contatto studiando l'attrito linguistico (fenomeno che consiste nella perdita totale o nel graduale venir meno della conoscenza della madrelingua) in contesti migratori dell'età infantile e pre-adolescenziale e dimostrando come nella memorizzazione linguistica l'età giochi un ruolo importante.

Pur presente in molti dei lavori, la città di Venezia è al centro dello studio di Slavko Petaković, che analizza il tema delle nozze a Venezia nell'epica serba, continuando una tradizione di studi della poesia orale dei Balcani particolarmente cara alla slavistica italiana. Similmente Vesna Kilibarda torna e scopre nuovi dettagli sul tema della visita di Petar Petrović Njegoš a Venezia. Due lavori di critica tematica si occupano del motivo del mare negli scrittori moderni della Jugoslavia: Svetlana Šeatovic analiza come due autori della terraferma, Ivo Andrić e Miloš Crnjanski, idealizzano la cultura del mare, mentre Rosanna Morabito segue il motivo del mare in un'opera meno nota dello scrittore bosniaco Meša Selimović, L'isola, del 1974.

Diversi lavori sono dedicati alla letteratura odeporica a partire dal ' 600 fino ai giorni nostri. Giovanna Moracci analizza il celebre Viaggio per l'Europa (I697-I69g) di Pëtr Andreevič Tolstoj proponendo un'analisi narratologica dei passi diegetici e descrittivi, dando una classificazione di questi ultimi in sottocategorie, e ponendo attenzione alla focalizzazione del narratore. Malgrado l'approccio segua primariamente il metodo strutturale, lo studio prende in esame anche imprescindibili aspetti storici, culturali, linguistici e religiosi che il viandante annotava o ai quali alludeva nel suo scritto. Della vivace personalità di Stefano Zanović (175I-1786), le cui strade si intersecano con quelle di Casanova, si occupa il lavoro di Persida Lazarević Di Giacomo dimostrando come la conoscenza dell'opera di Alberto Fortis avesse spinto Zanović a modificare il suo modo di scrivere e a sfruttare la sua provenienza geografica in maniera che si potrebbe dire auto-esotizzante. Nelle sue narrazioni coinvolgenti e al limite dell' inverosimile si profila la ricchezza di un secolo fatto di lumi e di illusioni, di verità e di immaginazione. Nicoletta Cabassi esamina il diario di bordo di un ufficiale della marina russa, Vladimir Bogdanovič Bronevskij, impegnato in una missione sul mare Adriatico nel I806, mentre Olivera Popović rilegge libri di viaggio, reportage ed articoli dei giornalisti italiani 
arrivati in Montenegro nel 1896 in occasione del fidanzamento di Vittorio Emanuele di Savoia con la principessa montenegrina Elena Petrović Njegoš, portando alla luce un corpus di testi piuttosto omogeneo in cui la creazione di una nuova immagine del Montenegro è evidentemente influenzata dal contesto politico. Questa nuova immagine va contro gli stereotipi romantici e balcanistici della terra primitiva di eroi e forti passioni e ne risalta al contrario i valori di modernizzazione, istruzione e sviluppo sociale. Giulia Baselica analizza uno sguardo russo sull'Italia del primo Novecento: le Immagini d'Italia (I9II) di Pavel Muratov, critico e storico dell'arte che scopre luoghi e monumenti meno noti ai viaggiatori russi che scrissero prima di lui. Infine, Ljiljana Banjanin presenta quello che è probabilmente il più recente diario della navigazione tra le coste adriatiche - opera dello scrittore triestino Mauro Covacich, che si presenta in forma ibrida tra reportage e letteratura di viaggio e contempla questioni di identità e alterità culturale, individuale e collettiva.

Dunja Kalođera e Ivana Tomić Ferić scrivono dell'illustre spalatino Julije Bajamonti, intellettuale illuminato della seconda metà del $\mathrm{I} 8 \mathrm{mo}$ secolo, testimone degli incontri tra le culture occidentali e orientali che si intersecano nell'Adriatico, e allo stesso tempo amico e corrispondente di numerose personalità europee. Il contributo si concentra in particolare sulla sua attività musicale, ed è corredato da un'appendice contenente trascrizioni di alcune sue importanti lettere e di riproduzioni dei suoi manoscritti.

Di letteratura dalmata in lingua italiana, riallacciandosi e ampliando gli studi pionieristici sul campo fatti da Mate Zorić, trattano i lavori di Sanja Paša e Boško Knežić. Sanja Paša presenta la poesia satirica poco nota dello spalatino Luigi Tommaseo, cugino di Nicolò, che scriveva in italiano, in dialetto veneto spalatino e in latino maccheronico, ma che nutrendo poca fiducia nel valore dei propri componimenti, non pubblicò quasi nulla della sua opera. La sua satira è fondata su un convinto antidogmatismo, anticlericalismo e fede nella razionalità umana, e prende di mira la situazione economica in Dalmazia, la mentalità e i costumi, mentre in dettaglio è qui descritta la sua polemica contro le dottrine cristiane in astronomia. Boško Knežić analizza invece i canti storici Le notti adriatiche di Luigi Fichert, in cui si intrecciano due aspetti del mare: da una parte quello immaginario ottocentesco, romantico, onirico, e dall'altra quello concreto e storico-politico. Fichert è un buon esempio di quel complesso mondo adriatico ottocentesco di intellettuali con sentimenti nazionali già ben definiti, ma non allineati alle idee politiche di omogeneità territoriale - un mondo per il quale la storica Dominique Kirchner Reill ha coniato la riuscita definizione di "nazionalisti che temevano la nazione".

Anche dopo questo tollerante ed aperto periodo ottocentesco, nelle nuove costellazioni di spinte nazionali a cavallo del ventesimo secolo, la stampa croata in Dalmazia ha mantenuto un forte interesse per la letteratura italiana: ne parla lo studio di Robert Bacalja e Antonio Sammartino, i quali nelle riviste croate dell'epoca registrano la presenza dominante di Carducci, Pascoli e D’Annunzio, e il complesso rapporto dei maggiori scrittori croati come Vladimir Nazor con questi classici italiani.

Decisamente diverso è il discorso jugoslavo tra le due guerre, come dimostra l'analisi di Marija Mitrović dedicata al fenomeno di Jadranska straža, un'associazione culturale di massa che si occupava della regione dalmata e della propaganda politica sotto il patrocinio del governo monarchico. Il movimento doveva rafforzare l'unitarismo jugoslavo fondandolo proprio sul posesso territoriale della costa dalmata e del suo mare, in concorrenza con l'Italia. Ma il grande slancio, la notevole attività editoriale e una forte ramificazione di sedi in tutto il territorio jugoslavo, non riuscirono a cambiare il sostanziale disinteresse della capitale Belgrado e dello stato centralizzato per la regione adriatica, per cui l'autrice del contributo alla fine concorda con lo scetticismo del poeta croato Tin Ujević, che nel 1930 constatava che per Belgrado la Dalmazia è "come se [...] non esistesse". 
Infine, Maria Rita Leto si è assunta il difficile compito di riesaminare la biografia e la bibliografia di Arturo Cronia e in particolare le sue note pagine influenzate dalla politica imperialista del ventennio e dalle sue inclinazioni personali. Sono pagine che avevano suscitato già da tempo alcune riserve degli studiosi, senza che si arrivasse, però, mai a negare al grande studioso l'enorme contributo dato alla slavistica e in particolare alla serbocroatistica italiana. Scegliendo e rileggendo le pagine e certe particolari espressioni di Cronia, Leto riesce a individuare degli intenti di superamento del proprio nazionalismo metodologico, magari non sempre consapevoli ("quasi suo malgrado"), ma certamente più comprensibili e più vicini ai modi di pensare e di fare ricerca dei giorni nostri. Torniamo così all'auspicio, espresso all'inizio da Egidio Ivetic, che nelle ricerche sull'Adriatico si utilizzino metodi e prospettive transnazionali, auspicio che può essere considerato il denominatore comune dell'intero volume.

L'Adriatico tra sogno e realtà conferma lo stretto rapporto che lega la slavistica italiana, l'italianistica dei paesi slavi (non solo quelli confinanti con le sponde del mare comune) e la comparatistica in generale al tema dell'Adriatico, aggiornandone le prospettive di studio e offrendo ai suoi lettori idee e spunti per future ricerche. 